\title{
Revue noire, une histoire
}

Entretien avec Monique Sicard et Claire Riffard

Jean Loup Pivin

\section{(2) OpenEdition}

1 Journals

Édition électronique

URL : https://journals.openedition.org/coma/433

DOI : $10.4000 /$ coma.433

ISSN : 2275-1742

\section{Éditeur}

Institut des textes \& manuscrits modernes (ITEM)

\section{Référence électronique}

Jean Loup Pivin, «Revue noire, une histoire », Continents manuscrits [En ligne], 3 | 2014, mis en ligne le 08 novembre 2014, consulté le 12 novembre 2021. URL : http://journals.openedition.org/coma/433 ; DOI : https://doi.org/10.4000/coma.433

Ce document a été généré automatiquement le 12 novembre 2021.

\section{(c) $(1) \odot$}

Continents manuscrits - Génétique des textes littéraires - Afrique, Caraîbe, dispora est mis à disposition selon les termes de la licence Creative Commons Attribution - Pas d'Utilisation Commerciale - Pas de Modification 4.0 International. 


\section{Revue noire, une histoire}

Entretien avec Monique Sicard et Claire Riffard

Jean Loup Pivin

Fig. 1 : Couverture du premier numéro de la Revue noire, 1991

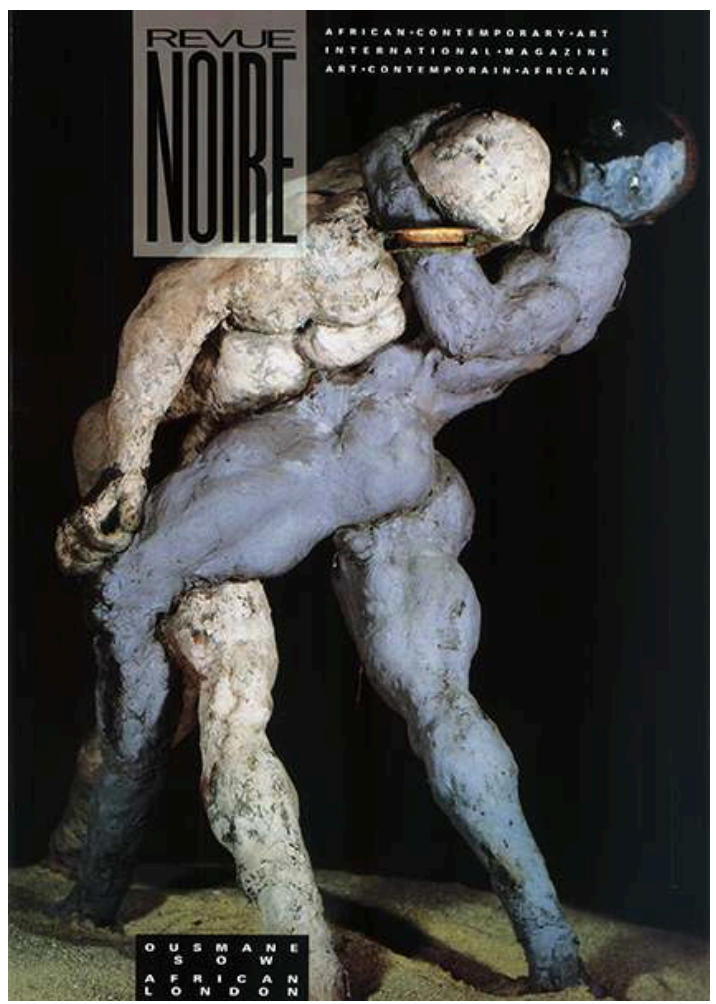

Photographe anonyme. Titre: Ousmane Sow : Couple de lutteurs corps à corps, Nouba, 1987. Dimensions de l'image : $27,9 \times 40 \mathrm{~cm}$. Fonds : Revue noire.

(c) Photo Revue noire 


\section{Préambule}

1 Le premier numéro de cette revue de grand format, somptueusement illustrée, date de l'année 1991 et s'intitule « Ousmane Sow / African London ».

2 Entièrement bilingue, Revue noire se donne pour objet de faire découvrir l'« African Contemporary Art / art contemporain africain ». Elle possède des correspondants dans toute l'Europe, en Amérique et en Afrique et en appelle à l'universel, à distance des réseaux francophones :

Peintres, sculpteurs, photographes, architectes, metteurs en scène, artistes, responsables de galerie, de musée, de centre culturel, d'association... des trois continents, tenez-nous informés de votre travail et de vos activités, pour faire de Revue Noire votre revue ${ }^{1}$.

3 Michel Leiris était mort l'année précédente, le 30 septembre 1990. Revue noire s'inscrit sous l'égide de l'un de ses textes ${ }^{2}$ et rend hommage à l'ancien Directeur de recherche au CNRS (par ailleurs, écrivain surréaliste et ethnographe membre de la mission DakarDjibouti (1931-1933), auteur de L'Afrique fantôme). Pour citer Revue noire, p. 5 : « Leiris fut sans doute l'un des premiers à considérer tous ces masques et ces objets sacrés ou païens, fonctionnels ou décoratifs, comme des œuvres d'art pleines et entières ». La revue donne longuement la parole à Leiris lui-même :

De même que chez d'autres peuples longtemps pris pour des "primitifs", la personne humaine, ses enveloppes et les divers accessoires mobiliers et immobiliers qui font partie de ses entours ont été - et sont encore fréquemment - chez les noirs africains le support ou le corps même d'un grand nombre de réalisations dont la qualité esthétique s'avère souvent très haute (...).

Toutefois, autant qu'autorise à en juger ce que l'on connaît des arts et métiers chez les peuples actuels de l'Afrique noire, il semble que dans cet immense territoire les activités plastiques, qui montrent de quelle somme considérable de savoir et d'habileté peuvent disposer des groupes relativement démunis, n'aient jamais tendu - ou n'aient tendu qu'exceptionnellement - à la production d' «œuvres d'art » au sens d'œuvres sans autre raison d'être que leur existence même (notion, d'ailleurs ignorée longtemps dans toutes les parties du monde).

Hormis celles qui, additions plus ou moins ornementales, ont un caractère à proprement parler « artistique » dans la mesure où elles jouent un rôle décoratif, le fait est que la plupart des œuvres africaines - tant profanes que sacrées - étudiées jusqu'à ce jour répondent à des besoins étrangers au domaine de l'art comme tel et qu'elles doivent être tenues pour fonctionnelles, à des titres variés. Pourtant, cela ne signifie nullement qu'elles ne comportent aucun élément de beauté pure, autrement dit : de beauté susceptible d'être goûtée pour elle-même. (...) estimer que le sentiment artistique comme tel est étranger aux Africains, fût-ce pour les louer d'être ainsi radicalement exempts de toute sophistication "esthète», cela ne reviendrait-il pas, d'ailleurs, à les priver encore une fois du statut d'hommes à part entière?

4 Près de 60 ans après la publication de L'Afrique fantôme, Françoise Huguier et Michel Cressole publient Sur les traces de l'Afrique fantôme ${ }^{3}$. On trouve également dans ce premier numéro un entretien avec Pierre Gaudibert ${ }^{4}$ :

La mondialisation de la culture crée des échanges d'images fortes dans lesquelles la production africaine est absente le plus souvent par méconnaissance. D'où l'urgence qu'il y a à organiser la promotion d'un art africain en pleine effervescence, à l'initiative même des principaux intéressés, qui éprouvent le besoin de voir les originaux, de travailler à Paris, et de s'y faire connaître. (...) (...) la faiblesse des marchés intérieurs et d'une façon plus générale, les difficultés 
économiques des pays africains ne laissent pas beaucoup d'espoir à un artiste dans son pays.

À travers cet état de choses, il faut noter une accélération des changements dans les cultures africaines qui s'ouvrent très fortement sur le monde: de nouveaux comportements naissent.

Plus on va dans les grandes métropoles où les relations communautaires sont déstabilisées, plus les individus sont à la recherche d'expressions artistiques multiformes (l'artiste sera souvent à la fois chanteur, peintre, danseur, poète...) liées à l'affirmation forcenée d'une individualité.

\section{Petite histoire de la photo en Afrique}

En inventant Revue noire, nous pensions qu'avant de faire la critique de l'art africain contemporain, il fallait en constituer le savoir, la connaissance. Constituer l'objet. L'Histoire de l'art, telle qu'on l'entend et dont nous sommes nous-mêmes nourris, est occidentale. C'est une idée occidentale qui a imprégné le regard que l'on posait sur les cultures "étrangères" dans le temps et dans l'espace comme l'art égyptien pharaonique ou sumérien. J'aime bien cette formule de Malraux: "Il n'y a plus maintenant d'art archéologique et d'art ethnographique, il n'y a plus que l'histoire des formes ». L'univers des formes. C'est bien le sens de notre travail.

Néanmoins Revue noire, c'est d'abord un refus ; c'est le refus de la critique construite sur l'histoire de l'art occidental. Et la volonté de découvrir avant tout, de découvrir des artistes. Nous en avons publié 3400 , dont plus de 600 photographes! Revue noire, c'est une découverte de la modernité africaine.

7 Si l'on voulait résumer l'histoire de la photographie en Afrique, on pourrait dire qu'à la grande époque, 1900-1960, celle dont il nous reste de nombreuses images, les photographes de studio étaient majoritaires et « autorisés » par la puissance coloniale. Puis dans les années 1950, la photo s'est libérée. Avec les Indépendances, les photographes comme Malick Sidibé à Bamako ou Jean Depara à Kinshasa sortent des studios et photographient les moments de bonheur dans les night club, les piqueniques à la campagne, au bord du fleuve ou de la mer. 
Fig. 2 : Série "Les Nuits et les jours de Kinshasa »

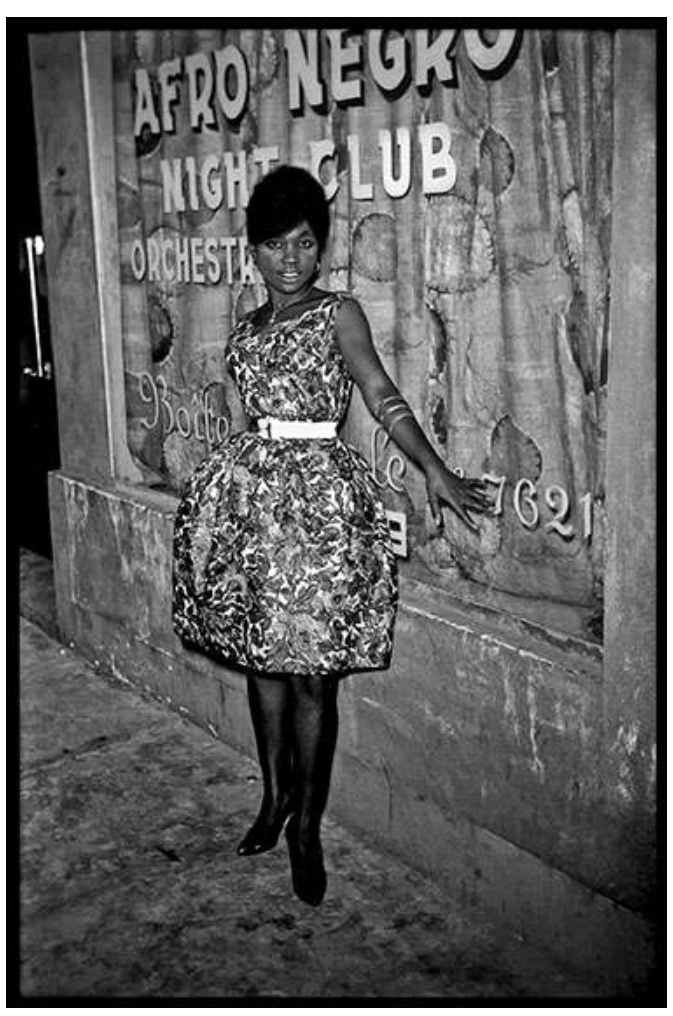

Auteur : Jean Depara (1928-1997)

(c) Revue noire

8 Ces années 1950-1960 sont l'époque des agences de presse, sur le modèle de l'Est pour les pays «non alignés », d'obédience soviétique puis chinoise, mais aussi pour les pays «bien tenus» par des autocrates comme le Zaïre de Mobutu. Ainsi se développent les images de propagande qui changeront de support avec l'arrivée de la télévision. La photographie de presse existera toujours, mais ce sera dans des conditions extrêmement difficiles quand le photographe n'est pas salarié.

9 Dès les années 1980-1990, les studios photographiques concurrencés par la vulgarisation des appareils $24 \mathrm{X} 36$ et les laboratoires automatiques couleurs, «mini labs ", disparaissent petit à petit. 
Fig. 3 : Jean Depara devant son studio

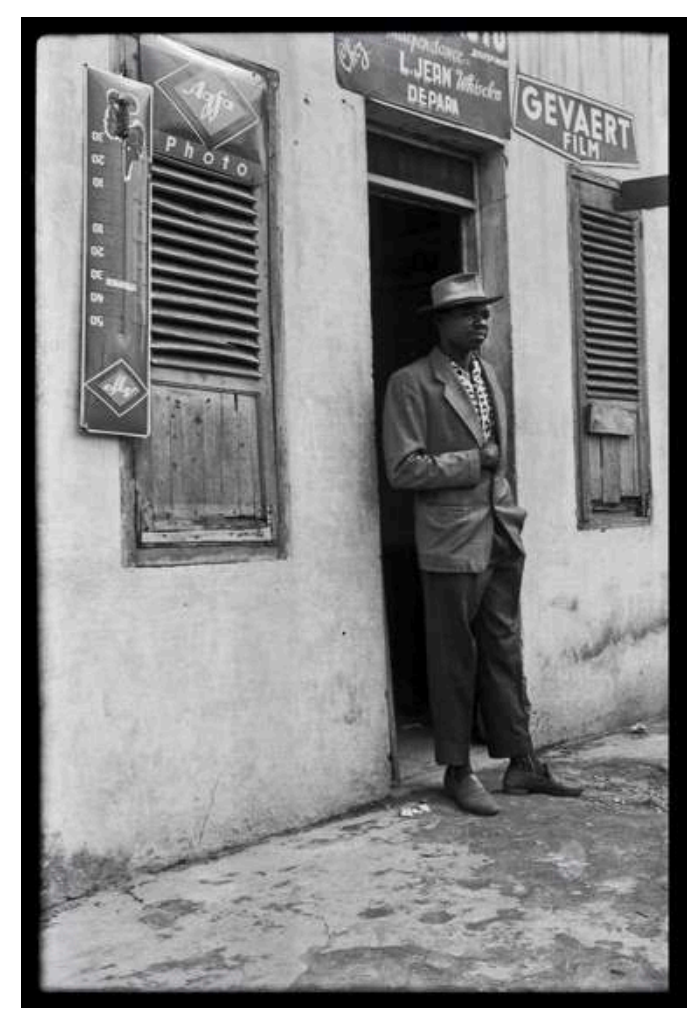

Photographe anonyme. Fonds : Revue noire.

(c) Revue noire

10 Un des rares débouchés reste alors la photo de publicité, comme en Côte d'Ivoire, et la photo de mariage, presque tout le temps associée à la vidéo. Le marché des ONG est réel mais incombe rarement aux photographes nationaux. Les ONG préfèrent les photographes européens, qui selon elles savent mieux quelles sont les motivations du public... occidental, pour déclencher son acte donateur. Le système est devenu pervers, ôtant à la photographie africaine sa principale qualité : celle de fabriquer des images dont l'esthétique correspond au désir de ses clients. Ces images commandées et payées par les Maliens, Ghanéens, Ivoiriens, Congolais, Nigérians... aux photographes de leur pays selon l'image qu'ils cherchent d'eux mêmes, petit à petit disparaissent. Certes pas totalement mais au profit d'une pratique amateur dont les images sont d'un autre ressort, plus intime. D'autant plus que les nouvelles technologies de plus en plus accessibles n'ont aucun retard en Afrique.

11 Au début des années 1990, le marché de l'art contemporain africain n'existait pas et encore moins celui de la photo africaine puisque personne ne la connaissait. Les professionnels occidentaux disaient même que les photographes africains n'existaient pas. Quant à la problématique du «marché » de la photographie d'une façon générale, elle était encore balbutiante. Vivre de la vente de ses tirages comme d'œuvres d'art n'est pas dans les préoccupations de tous les photographes du globe.

Fig. 4 : Tirage au studio Depara 


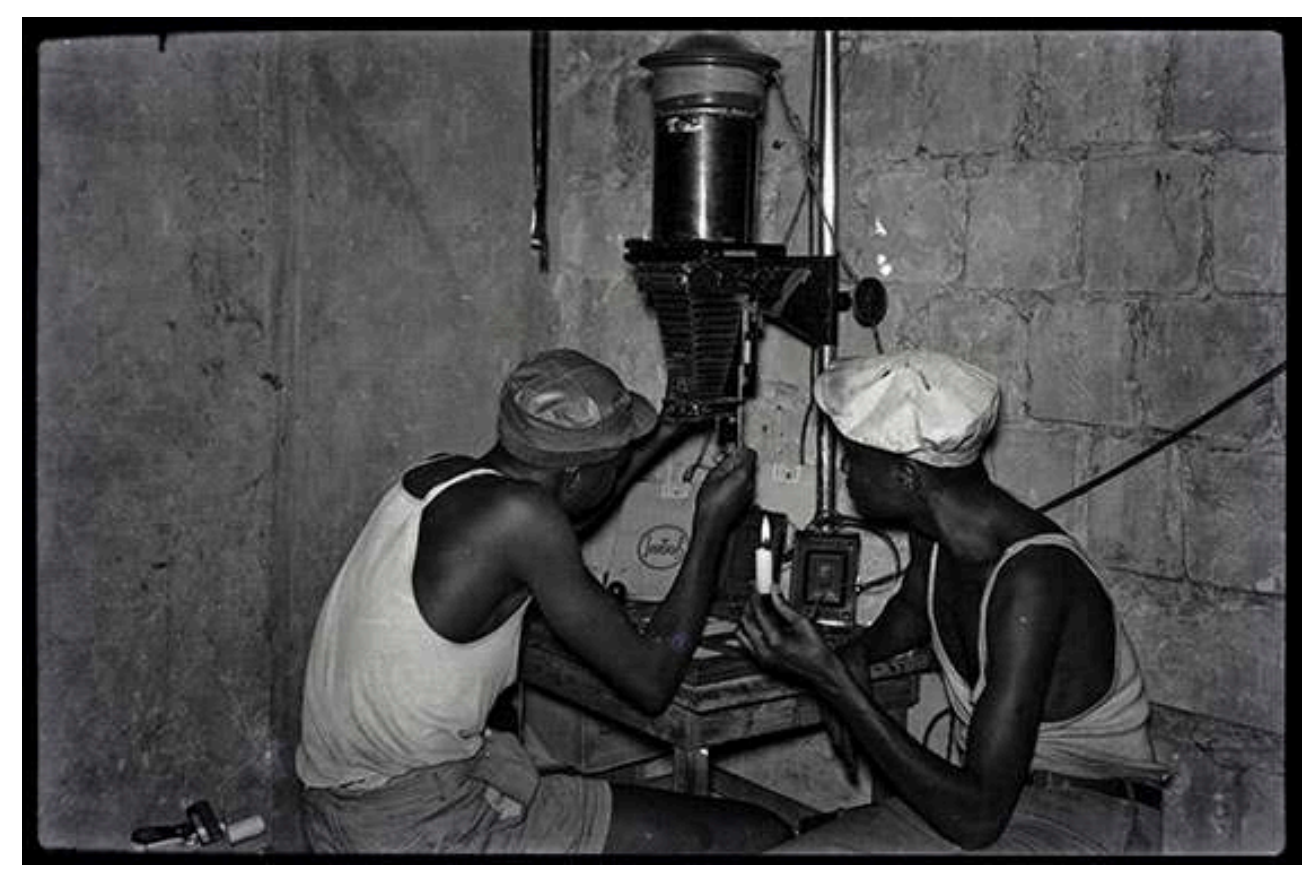

Photographe anonyme. Fonds : Revue noire.

(C) Revue noire

12 C'est dans ce cadre que nous avons publié nos premiers numéros de Revue noire, montrant d'entrée de jeu une préoccupation à rendre manifeste l'existence de ces photographes. A chaque numéro de Revue Noire, nous cherchons, nous enquêtons, nous faisons le tri, nous retrouvons des archives au milieu de fouillis. Parallèlement, des photographes européens, lors de leurs voyages en Afrique, faisaient des rencontres de photographes africains qui se révélèrent par la suite de véritables « découvertes ». Ainsi Françoise Huguier, qui était « sur les traces de l'Afrique fantôme » - c'est le titre d'un de ses ouvrages en hommage à Michel Leiris rencontre Malick Sidibe en cherchant un réparateur photo à Bamako !

13 À Madagascar, après avoir consulté et publié les archives des deux agences ANTA ${ }^{5}$, nous avons réalisé une enquête avec le photographe Dany Be et un groupe de ses amis sur l'histoire de la photo à Madagascar.

14 Les premières photos prises par des Malgaches datent d'avant 1900. A L'Île Maurice, il y a une tradition photographique depuis 1848. En Egypte, c'est le Sultan lui-même qui est le premier photographe « oriental » dès les années 1970. On néglige souvent le fait que la photographie de ces pays n'est pas uniquement faite par les voyageurs et les studios d'Européens installés sur place. Et les découvertes se multiplient, même si nous regrettons que cette préoccupation se fasse jour si tard. À la période même où nous entamons la publication de photographes africains, de nombreux photographes importants sinon essentiels meurent comme Mama Casset au Sénégal, Amichia et Cornelius August Azaglo en Côte d'Ivoire ou Depara en RDC/Zaïre. Les archives photographiques de Cornelius ont été sauvées par Jean-François Werner et conservées à l'IRD ${ }^{6}$. Mama Casset et Amichia ne laissent aucun négatif, tandis que quelques centaines de Depara sont sauvés au détriment des dizaines de milliers d'autres détruits. 
Fig. 5 : Studio du photographe Tennquin

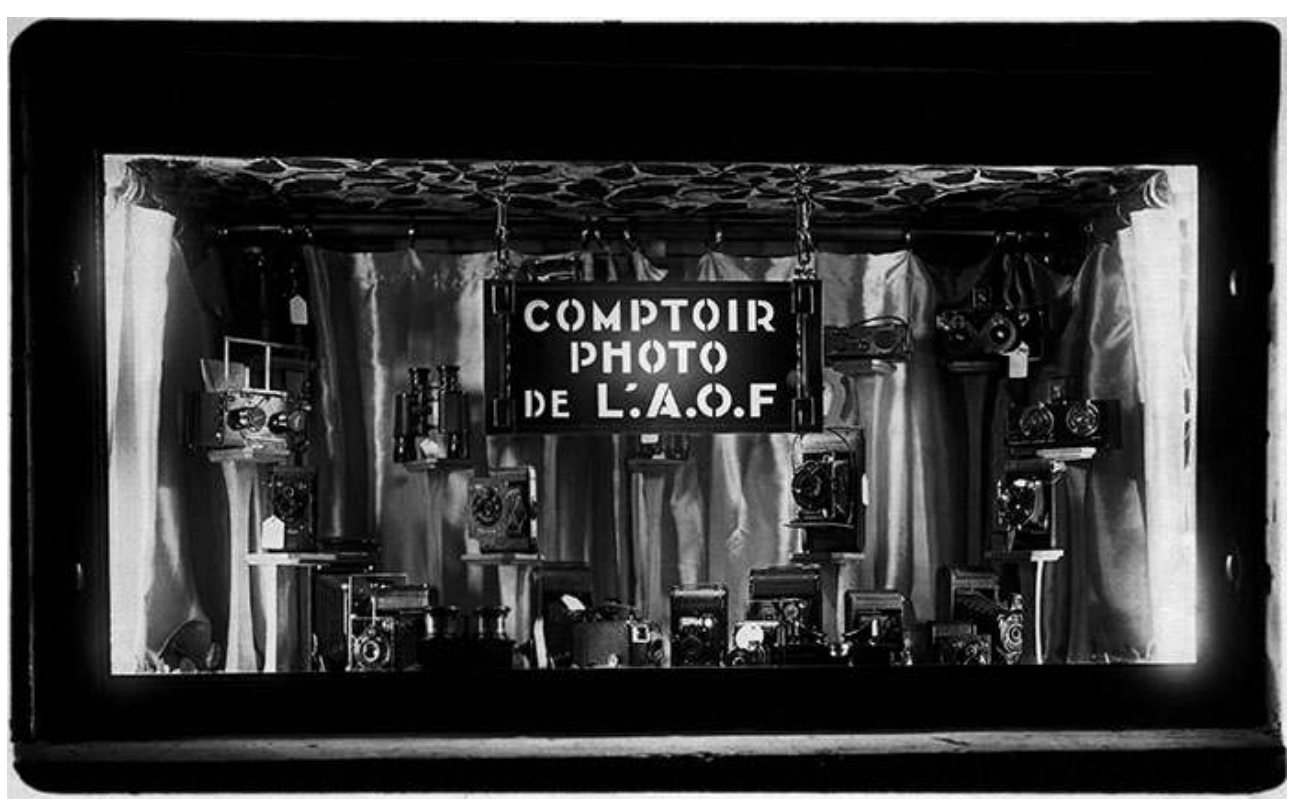

Photographe anonyme. Fonds : Revue noire.

(c) Revue noire

15 Si le propos de Revue noire exclut l'exotisme et la folklorisation de l'Afrique, aucun champ n'est exclu pour la photographie elle-même. Le sujet est trop neuf. Et c'est en cela que l'Anthologie de la Photographie liée à l'exposition "l'Afrique par elle-même " ${ }^{8}$ montre l'intégralité du spectre photographique: du studio au reportage, de l'art à l'essai, de la photographie de propagande à la photographie engagée et ce, sur une période allant de 1900 à nos jours.

Alors que les premiers pas de la reconnaissance d'une photographie africaine se dessinaient, deux noms perçaient au détriment de tous les autres : ceux des Maliens Seydou Keita et Malick Sidibé, car probablement le marché ne pouvait pas en intégrer plus et le renvoi exotique fonctionnait totalement. À l'opposé, pendant les dix années de publications de Revue noire, 650 photographes africains seront publiés, couvrant l'ensemble du spectre de la pratique de la photographie en Afrique.

\section{Petite histoire de la Revue noire}

16 Nous avons concrètement démarré en 1989, avec une exposition qui n'a jamais été montée, «Dans la ville noire ». Elle devait être installée en même temps que la grande exposition de Jean-Hubert Martin "Magiciens de la terre ". Mais il n'y avait pas la place pour deux expositions en même temps sur l'Afrique. Alors que les Magiciens traitaient du monde sur le mode d'un art ne venant pas d'élèves d'école des Beaux Arts, nous voulions traiter les expressions contemporaines de la ville moderne africaine. Le projet a été annulé. Revue noire est née de la colère de l'impuissance et de notre désir de sortir ce continent d'un angélisme coupable visant toujours à l'altérité et le montrer sous l'angle du partage de destin.

Nous avons décidé à ce moment de poser, lourdement!, la première pierre d'une "histoire de la photographie africaine». Le projet est monté au niveau mondial ${ }^{10}$. L'exposition s'appelle "l'Afrique par elle-même ", et le catalogue "Anthologie de la 
photographie africaine et de l'océan Indien ». Nous sommes passés au bord du gouffre financier! Et Pascal Martin Saint Leon et moi-même avons converti le béton des quelques biens que nous avions en papier ; c'est l'inverse de la pratique des architectes que nous étions, un comble!

Évoquer l'argent quand on est éditeur est fondamental, car dire cela c'est dire l'engagement que nous avions, associé à la liberté qui en découlait. Même si par la suite (à partir du $\mathrm{n}^{\circ} 4$ ) nous avons reçu des aides publiques - essentiellement du Ministère de la Coopération devenu Affaires Etrangères - et de l'Union Européenne, elles n'ont jamais couvert ne serait-ce la moitié de ce que Revue noire coûtait. Sans parler du bénévolat de chacun des fondateurs Simon Njami, Pascal Martin Saint Leon, Bruno Tilliette et moi-même.

Cette distance avec les institutions publiques nous permettait néanmoins de ne répondre à aucune demande ou même proposition venant d'elles si nous n'étions pas intimement convaincus de leur bien-fondé. Nous ne nous cassions pas la tête en nous ruinant pour aller là où on nous disait d'aller! D'où la " presque » non-existence de livres de commande dans le catalogue de Revue noire, à la différence de la quasi totalité des éditeurs d'art français. De la même façon le bilinguisme absolu de nos publications était une façon de renoncer à cette dichotomie néocoloniale des prés carrés. En choisissant "Londres » comme thématique pour le $\mathrm{n}^{\circ} 1$, nous faisions de Revue noire un symbole de cette distance par rapport à la problématique de la francophonie. Dans les derniers temps de la revue, nous diffusions plus lourdement dans les pays anglophones (5000 exemplaires) que dans les pays francophones (3000 exemplaires).

Nous voulions capter l'art en train de se faire. Nous avons passé commande à des photographes, en leur donnant des pellicules et 100.000 francs CFA (150€), et nous avons attendu les résultats. Ainsi, à chaque numéro, la photographie contemporaine s'exprimait. Parfois, nous leur disions « Donnez-nous l'image de votre vie ». Nos choix étaient très anticonformistes. Nous allions parfois vers des photos floues car elles nous plaisaient. Ça a sans doute créé une déformation du marché: tout le monde nous apportait des photos floues!

Nous passions commande, de pays en pays. Mais l'effet retombait tout de suite après notre passage, car les photographes ne travaillent que s'ils vendent.

Peut-on parler de "photographie africaine »? La situation de la photographie est très différente de celle des autres arts. Les arts plastiques «modernes» sont souvent issus de l'artisanat et des différents centres artisanaux créés pendant la colonisation; ils avaient pour principal marché le colon, le voyageur occidental, aujourd'hui le touriste, le coopérant et les collectionneurs d'art contemporain. Le théâtre a lui aussi été touché par une politique post coloniale pour le moins ambiguë, réduisant le public local au profit des tournées européennes et américaines. Par contre, la photographie n'a pas été touchée originellement par ce phénomène de public exogène car elle a toujours été destinée au marché africain, et non au marché occidental ; elle a eu cette liberté-là, comme la création textile. Et ce jusqu'à ces dernières décennies où la vente en galerie de la photo en tant qu'art en a fait de nouveau un produit destiné au marché extérieur à l'Afrique, pour ne pas dire occidental.

Pour autant, peut-on parler d'une photographie africaine? On ne peut certainement pas dire qu'il y ait un "style africain", dont la caricature serait la photographie de studio d'Afrique de l'Ouest. Mais par contre, parler de la photographie faite par des 
Africains veut réellement dire quelque chose, avec probablement une spécificité des sujets photographiés par les photographes africains, qui n'ont rien de commun avec l'Afrique photographiée par les voyageurs européens. Ce qui fait que l'on peut parler par facilité de photographie africaine. Et en ce sens, on devrait d'ailleurs mettre l'expression au pluriel et parler des photographies africaines.

Quant à nous, nous avons toujours voulu privilégier la modernité de la photo africaine en laissant la caricature de côté, y compris lorsqu'il s'agissait de photographes de studio. Si vous observez le travail des photographes de studio, qui par centaines et milliers se sont développés jusque dans la moindre petite ville, vous retrouvez souvent les mêmes décors que ceux de Seydou Keita, et les mêmes poses. Sachant cela, nous avons lancé une grande enquête que nous avons confiée à un photographe ivoirien, Dorris Kasco Haron, dont nous avions déjà publié une série sur les fous d'Abidjan. Plus de 200 photographes de studio ont été ainsi sortis de l'oubli, dont Azaglo et Amichia pour la Côte d'Ivoire.

L'exemple de Madagascar est intéressant. Qu'est-ce qu'on connaît, qu'est-ce qu'on ignore de la photographie malgache? Les archives des photographes officiels, maintenant, on les connaît. Mais il faudrait faire une enquête sur les photographes de quartier.

Si la photographie malgache nous a beaucoup intéressés, c'est parce qu'elle joue avec les codes européens. S'il y a bien un monde ouvert aux mondes européens, qui n'a jamais eu de complexe face à l'Europe, c'est bien Madagascar. Regardez l'écrivain Rabearivelo et la manière dont il posait habillé avec élégance. Ce n'est pas un hasard si le Palais de la Reine, à Tana, a été dessiné par un Français. Même les maisons dites «traditionnelles » des hauts plateaux sont le résultat d'un syncrétisme, ce n'est pas la case originelle, la paillotte, ce sont des maisons avec véranda. En ville, à l'époque, les dames de la bourgeoisie malgache portent des ombrelles!

La photographie malgache est le fait de cette bourgeoisie malgache, qui adopte les codes et les attributs européens. Et qui a du goût! Le cristal de Baccarat de leurs lustres, c'est du vrai, pas du toc!

En photographie, cela produit un travail comme celui réalisé par Ramily sur les paysages, très éloigné du travail en studio. Ramily était un très bon tireur qui a formé tous les photographes de la génération de Pierrot Men. Et il vendait! Qui n'avait pas chez lui une photo de Ramily?

Ce lien entre photo et bourgeoisie existe dans tous les pays africains. Salaky, pour ne parler que de lui, venait d'une famille de marchands marocains implantée à SaintLouis du Sénégal. Il est arrivé au Mali avec le train Dakar-Bamako, pour y implanter son studio, un commerce comme un autre.

On pourrait même aller jusqu'à faire le parallèle avec l'introduction des armes en Afrique. L'histoire de la photo, c'est comme l'histoire des armes. Elle est perçue comme un progrès évident et adoptée par les gens pour des raisons pratiques. C'est le même processus que de mettre de la tôle sur le toit de sa baraque : on entre dans la modernité, on entre dans le temps.

Les photographes choisissent des éléments de la modernité pour être de leur temps.

Fig. 6 : Sans titre, série "African Studio" 


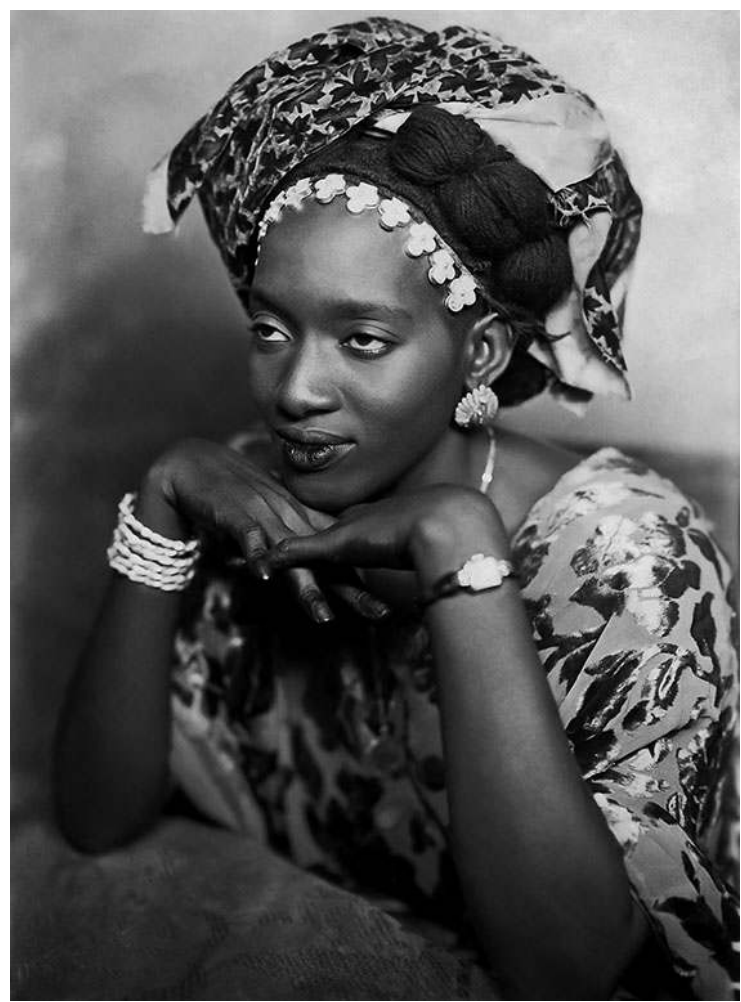

Mama Casset (1908-1992). Fonds : Revue noire.

(c) Revue noire

21 L'une des grandes influences pour les photographes africains d'Afrique de l'Ouest a été celle de Mama Casset. Ses postures. Sa sensualité. Et probablement aussi le fait de vendre des photographies qui ne sont pas forcément celles de famille. Formé par un photographe français de Saint Louis au début $\mathrm{du} \mathrm{XX}^{\circ}$ siècle, il a très vite développé son style unique, qui n'aurait pas existé sans les séduisantes, puissantes et riches SaintLouisiennes et signares. Les quelques images qui restent de son studio (qui brûla dix ans avant sa mort), montrent cette immense qualité qui forme un style, avec ce jeu de mains, le regard au loin qui donne une distance aristocratique, inaccessible et pourtant si sensuelle. Tous les photographes, à commencer par son frère mais aussi Sakaly et Keita évoqués pour le Mali, connaissaient son travail que l'on pouvait voir dans la vitrine de sa boutique. Même les peintres de souwères ${ }^{11}$ reprenaient les images de ces belles sénégalaises prenant la pose.

Faut-il faire entrer la photographie africaine dans le marché international de la photographie?

Ce n'est peut-être pas le problème. On ne sait pas réellement pourquoi sont entrés sur ce marché international des photographes comme Malick Sidibé ou, bien plus incompréhensible encore, Ojeikeré. En quoi ces photographes populaires participent-ils d'une façon un tant soir peu consciente à cet art contemporain si codé? Il n'existe aucun exemple de photographe de même nature, c'est-à-dire populaire et des années 1960, en Europe, aux USA, au Japon ou en Chine, qui ait fait la moindre entrée dans l'art contemporain de ces vingt dernières années. Tout se passe comme si, pour l'Afrique, la caricature du «bon sauvage » était toujours de mise. Alors qu'il existe sur le continent de nombreux photographes dont le travail pourrait entrer dans cet art contemporain, comme Samy Baloji, Alain Polo et mieux encore le plasticien Joël Andrianomearisoa. 
C'est vrai que les photographes de studio et ceux des virées dansantes des années 1960 sont bien loin. Mais nous serions réellement dans le sujet. De même, David Goldblatt qui a incarné avec bien d'autres la lutte contre l'apartheid en Afrique du Sud est le seul qui soit lui aussi choisi par l'art contemporain. Pourquoi lui et pas un autre tout aussi talentueux ? L'art contemporain, trop lié à un marché, ne peut "assimiler » plus de quelques photographes - trois, quatre ou cinq. C'est en cela que les achats de la Tate Modern ou de grandes institutions d'art contemporain ou encore de collectionneurs suiveurs obéissent plus à des lobbys qu'à de véritables choix. Mais c'est un épiphénomène pour les milliers d'autres photographes africains. C'est pour cela que ce marché de l'art contemporain ne devrait pas être la seule ligne d'horizon vers laquelle les photographes africains regardent. Correspondre à une image que le "marché international » intégrera est pusillanime et stérile. Si cela se fait, tant mieux, sinon la pratique - qui peut être amateur - et le métier de photographe doivent se nourrir sur place, idéalement avec un public local, pour fabriquer des images qui, sans être nécessairement autonomes des grands courants, satisferaient un marché local de publicité, de mariage, de presse, et de recherche - aidée ou pas.

Fig. 7 : Couverture nu numéro 3 de la Revue noire, 1991

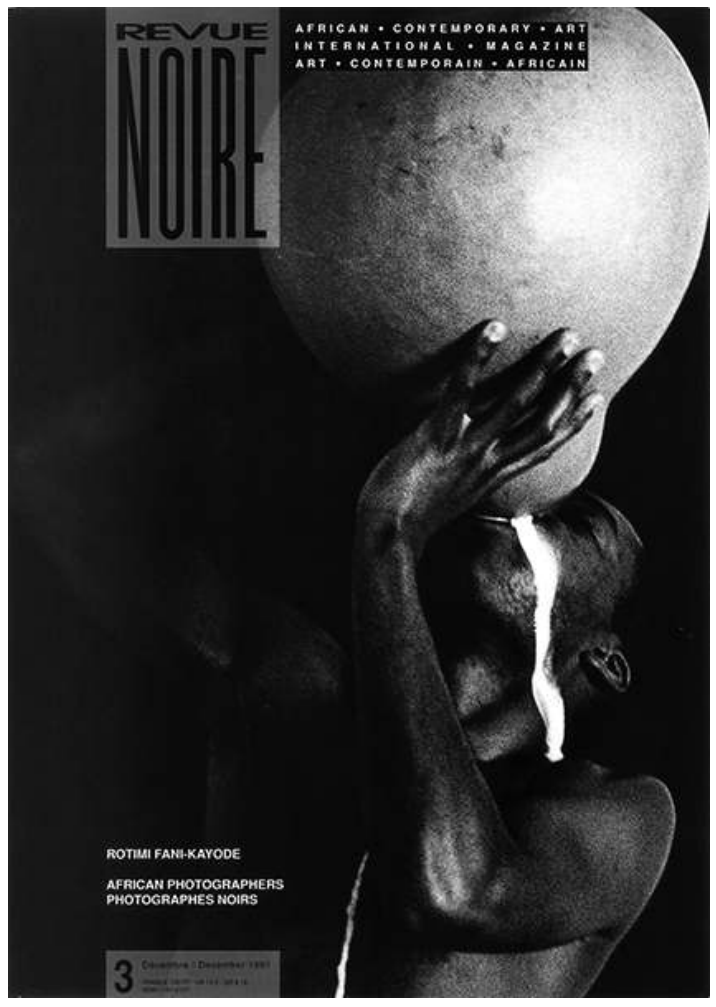

Rotimi Fani-Kayode. Titre: "Milk Drinker". Dimensions de l'image : 27,9 x $40 \mathrm{~cm}$. Fonds :Revue noire. (c) Revue noire 


\section{NOTES}

1. Revue Noire $n^{\circ} 1,1991$, p. 4.

2. L'Afrique noire, coll. «L'univers des formes », Éditions Gallimard, Paris, 1967, cité en page 4 de Revue Noire $\mathrm{n}^{\circ} 1$.

3. Françoise Huguier (photographies) et Michel Cressole (textes), Sur les traces de l'Afrique fantôme, Editions Maeght, Paris, 1990.

4. Conservateur en chef du patrimoine, Pierre Gaudibert s'est tourné dès les années 1970 vers l'art africain traditionnel et contemporain, ce qui participe d'un grand tournant de son regard sur le monde des formes. Le fait que les expressions africaines soient touchées par la foi voire par les Dieux remet en question un regard qu'il voulait jusque là matérialiste et marxiste. Grande figure de l'art contemporain, créateur de l'ARC au Musée d'Art Moderne de la Ville de Paris, il est conquis par les formes d'un continent qui iront jusqu'à son initiation au vaudou (culte fon yoruba) au Bénin.

5. Agence Nationale d'Information de Madagascar.

6. Institut de Recherche pour le Développement. Voir l'article de JF Werner dans ce même dossier.

7. Anthologie de la Photographie Africaine et de l'Océan Indien [200 portfolios de photographes africains à travers 500 photographies N\&B et couleurs, essais de 35 spécialistes du monde entier], sous la direction de Pascal Martin Saint Leon, N'Goné Fall, Jean Loup Pivin, Paris, Revue Noire, 432 pages $24 \times 32 \mathrm{~cm}, 1998$.

8. Exposition créée au printemps 1998 par Jean Loup Pivin et son équipe à la Maison Européenne de la Photographie de Paris, à partir de la note d'intention suivante : " "L'Afrique par elle-même" est une première exploration globale de la photographie d'Afrique non méditerranéenne et de l'océan Indien. Elle ne cherche pas à définir ou à inventer une photographie africaine. Bien au contraire, toutes les pratiques et esthétiques à travers le temps et l'espace sont présentées comme un grand collage, sans autre présupposé de style ou d'identité particulière. Cette exposition et la publication qui l'accompagne, regroupent l'ensemble des recherches et enquêtes faites aujourd'hui sur la photographie africaine, principalement celles de Revue Noire.»

9. A Paris, à la Villette.

10. Tournée de la Maison Européenne de la Photo de Paris jusqu'à la Smithsonian de Washington, en passant par la Biennale de Sao Paulo et le musée de Tervuren qui a conclu l'itinérance au bout de sept ans.

11. La peinture souwère est une technique traditionnelle de peinture, où l'artiste peint littéralement "sous le verre ", en appliquant la peinture à l'envers d'une plaque de verre, par couches successives.

\section{AUTEUR}

\section{JEAN LOUP PIVIN}

Fondateur et directeur de publication de Revue noire 